\title{
ITERATED FLOOR FUNCTION, ALGEBRAIC NUMBERS, DISCRETE CHAOS, BEATTY SUBSEQUENCES, SEMIGROUPS
}

\author{
AVIEZRI S. FRAENKEL
}

\begin{abstract}
For a real number $\alpha$, the floor function $\lfloor\alpha\rfloor$ is the integer part of $\alpha$. The sequence $\{\lfloor m \alpha\rfloor: m=1,2,3, \ldots\}$ is the Beatty sequence of $\alpha$. Identities are proved which express the sum of the iterated floor functional $A^{i}$ for $1 \leq i \leq n$, operating on a nonzero algebraic number $\alpha$ of degree $\leq n$, in terms of only $A^{1}=\lfloor m \alpha\rfloor, m$ and a bounded term. Applications include discrete chaos (discrete dynamical systems), explicit construction of infinite nonchaotic subsequences of chaotic sequences, discrete order (identities), explicit construction of nontrivial Beatty subsequences, and certain arithmetical semigroups. (Beatty sequences have a large literature in combinatorics. They have also been used in nonperiodic tilings (quasicrystallography), periodic scheduling, computer vision (digital lines), and formal language theory.)
\end{abstract}

\section{INTRODUCTION}

For a real number $\alpha$, the integer part or floor function $\lfloor\alpha\rfloor$ of $\alpha$ is the largest integer $\leq \alpha$. The (homogeneous) Beatty sequence of $\alpha$ is the sequence $S(\alpha, 0)=\{\lfloor m \alpha\rfloor: m=1,2, \ldots\}$, though the domain may often be taken as some infinite integral interval other than the positive integers. For any integer $m$, let

$$
A^{0}(m)=m, \quad A(m)=\lfloor m \alpha\rfloor, \quad A^{i}(m)=A^{i-1}(A(m))=A\left(A^{i-1}(m)\right) .
$$

In $\S 2$ we prove an identity which expresses a sum of the iterated functional $A^{i}$ for $0 \leq i \leq n$, operating on an algebraic number $\alpha$ of degree $\leq n$, in terms of only $A^{1}(m)$ and $A^{0}(m)$ and a term bounded in $m$. Such an identity, for the single number $\phi=(1+\sqrt{5}) / 2$ (the golden mean), has been proved and used recently $[19,38]$ for a number of investigations. In $\S 3$ we give an application to "discrete chaos", namely, we construct sequences with bounded discrete range, shown to' be aperiodic and dense in one of the cases. This in itself leads to two further directions: (i) Investigation of a special case in which the sequences are periodic with period 1, i.e., regular behavior ("discrete order"). This is taken up in $\S 4$. (ii) Explicit construction of infinite nonchaotic, i.e., regular subsequences of chaotic sequences, considered in $\S 5$.

A sequence of the form $S(\alpha, \gamma)=\{\lfloor m \alpha+\gamma\rfloor: m=1,2, \ldots\}$ with real modulus $\alpha>0$ and real residue $\gamma$ is a Beatty sequence. There is a large literature on Beatty sequences, e.g. $[2,46,18,12,24,39,49]$. Among other

Received by the editors July 19, 1991 and, in revised form, November 12, 1991. $58 \mathrm{~F} 99$.

1991 Mathematics Subject Classification. Primary 05A19, 11B83; Secondary 11B37, 11S05, 
applications and connections we mention Penrose tilings [35, 36, 29], periodic scheduling [48], formal language theory [5, 31] and computer vision. One of the questions arising in the latter area is: given a (usually finite) sequence of integers, are there $\alpha$ and $\gamma$ such that $T$ is (the prefix of) $S(\alpha, \gamma)$ ? This spectrum question (terminology due to Graham, I think) has been treated independently in the mathematical literature $[23,6,7]$ and in the computer science literature $[40,31,9,28]$, in fact, using different methods. A. Bruckstein told me that $\mathbf{M}$. Werman has "made the connection" between these parallel independent lines of investigations. (In fact, Werman had heard my lectures on these topics at the Weizmann Institute and later worked in computer vision.)

A disjoint covering system (DCS) is a finite collection of Beatty sequences which partitions the positive integers. It is an integer DCS if all its moduli are integers, in which case we write it as $\bigcup_{i=1}^{m} S\left(a_{i}, b_{i}\right)$ with $-a_{i}<b_{i} \leq 0$ $(1 \leq i \leq m)$; a rational DCS if all its moduli are rational; and an irrational DCS if all its moduli are irrational. Using nonelementary methods, Mirsky, Newman, Davenport, and Rado, proved that the two largest moduli of an integer DCS are equal (see [11]). For an elementary proof see [3].

Proposition I. For $\alpha>0$ irrational, $\{s(\alpha, \gamma), s(\beta, \delta)\}$ is a DCS if and only if

$$
\frac{1}{\alpha}+\frac{1}{\beta}=1, \quad \frac{\gamma}{\alpha}+\frac{\delta}{\beta}=0 ; \quad m \beta+\delta=K \quad(m, K \text { integers }) \Rightarrow m \leq 0 .
$$

See $[13,2,43,44,1,22]$. We conjectured that for every DCS $\bigcup_{i=1}^{m} S\left(\alpha_{i}, \beta_{i}\right)$ with $m \geq 3, \alpha_{i} / \alpha_{j}$ is an integer for some $i \neq j$, and that in fact $\alpha_{i}=\alpha_{j}$ except for the case $\alpha_{i}=\left(2^{m}-1\right) / 2^{m-i} \quad(i=1, \ldots, m)$ [14]. For special cases of the conjecture with rational DCS see [33, 4, 41, 42, 10]. Graham [22] has proved that for irrational DCS, $m \geq 3$ implies $\alpha_{i}=\alpha_{j}$ for some $i \neq j$, so the conjecture holds for integer and irrational DCS, but is curiously still open for rational DCS. Graham's proof consists of showing that for $m \geq 3$ every irrational DCS has a "kernel" consisting of some $\{S(\alpha, \gamma), S(\beta, \delta)\}$ satisfying the hypothesis of Proposition I, and all the moduli are integer multiples of $\alpha$ and $\beta$, so the result follows as for integer DCS.

We define a Beatty subsequence $S(\zeta, \eta) \subset S(\alpha, \gamma)$ to be nontrivial if $\zeta / \alpha$ is irrational, trivial if $\zeta / \alpha$ is an integer. In view of Graham's result, it is natural to ask whether nontrivial Beatty subsequences exist. Using the basic identity of $\S 2$ we demonstrate in $\S 6$ existence of nontrivial subsequences of $S(\alpha, 0)$ for an infinite class of irrational $\alpha, 1<\alpha<2$.

For $1<\alpha<2, S(\alpha, 0)$ consists of short blocks of $\lfloor\beta\rfloor-1$ consecutive integers and long blocks of $\lfloor\beta\rfloor$ consecutive integers, where $\alpha^{-1}+\beta^{-1}=1$. What is the precise location of all the long blocks in $S(\alpha, 0)$ ? Our methods permit to answer this question for the same infinite family of $\alpha$, as well as to show precisely, for this family, when the complement of a Beatty subsequence with respect to a Beatty sequence is itself a Beatty sequence ( $\$ 6)$.

The peculiar multiplication rule $k \times l=(3 / 2) k l$ is associative but does not always produce an integer for integers $k$ and $l$. If we force the result to be an integer, i.e., by writing $k \times l=\lfloor(3 / 2) k l\rfloor$, we no longer have an associative operation. For example, $(3 \times 5) \times 7=231$, while $3 \times(5 \times 7)=234$. It would seem exceptional to find associativity in operations whose definition involves truncation. In the final $\S 7$ we exhibit several such exceptional cases. We also 
exhibit affine mappings which preserve pairs of the form $(A(m), B(m))$ where $B(m)=\lfloor m \beta\rfloor, \alpha^{-1}+\beta^{-1}=1$. In both cases we generalize but a small sample of results about operations on the golden mean in $[19,38]$ to similar operations on certain infinite classes of algebraic numbers, using the basic identity of $\S 2$. We close by indicating some directions for further work.

Note that the basic identity of $\S 2$ is used both for exhibiting chaotic behavior (in $\S 3$ ) and to prove regularity properties of algebraic numbers $(\S \S 4-7)$.

We denote by $\mathbb{Z}, \mathbb{Z}^{0}$ and $\mathbb{Z}^{+}$the set of integers, nonnegative integers and positive integers respectively. Also $\{\alpha\}=\alpha-\lfloor\alpha\rfloor$ is the fractional part of $\alpha$.

\section{ITERATED FLOOR FUNCTION AND ALGEBRAIC NUMBERS}

Theorem 1. Let $n \geq 1$ and $a_{0}, \ldots, a_{n}, m, K, L, M \in \mathbb{Z}$. Suppose that

$$
a_{n} x^{n}+a_{n-1} x^{n-1}+\cdots+a_{1} x+a_{0}=0
$$

has a real nonzero root $\alpha$. Let $A(m)=\lfloor m \alpha\rfloor$. Then

$$
A\left(M+L m+\sum_{i=0}^{n-2} A^{i}\left(K a_{i \dot{ } 2} A(m)\right)\right)=\left(L-K a_{1}\right) A(m)-K a_{0} m+D,
$$

where $D$ is bounded in $m$, namely,

$$
\begin{gathered}
D=\left\lfloor M \alpha+\left(L+K a_{0} \alpha^{-1}\right)\{m \alpha\}-\theta \alpha\right\rfloor, \\
\theta=\sum_{i=1}^{n-2}\left(K a_{i+2} A(m) \alpha^{i}-A^{i}\left(K a_{i+2} A(m)\right)\right) .
\end{gathered}
$$

Proof. Let $k \in \mathbb{Z}, l \in \mathbb{Z}^{+}$. The boundedness of $D$ follows directly from

$$
0 \leq k \alpha^{l}-A^{l}(k)<\sum_{i=0}^{l-1} \alpha^{i}
$$

The left-hand side is clear. We use induction on $l$ (for every $k$ ) for the righthand side. For $l=1, k \alpha-A(k)=\{k \alpha\}<1$. Suppose it holds for $l$. Then

$$
\begin{aligned}
k \alpha^{l+1}-A^{l+1}(k) & =(A(k)+\{k \alpha\}) \alpha^{l}-A^{l}(A(k)) \\
& \leq\{k \alpha\} a^{l}+\sum_{i=0}^{l-1} \alpha^{i}<\sum_{i=0}^{l} \alpha^{i}
\end{aligned}
$$

Denote by $R$ the right-hand side of (2). Then

$$
\begin{aligned}
R & =\left(L-K a_{1}\right) A(m)-K a_{0} m+\left\lfloor M \alpha+\left(L+K a_{0} \alpha^{-1}\right)(m \alpha-A(m))-\theta \alpha\right\rfloor \\
& =-K a_{1} A(m)+\left\lfloor(M+L m) \alpha+K A(m) \sum_{i=0}^{n-1} a_{i+1} \alpha^{i}-\theta \alpha\right\rfloor \\
& =\left\lfloor\left( M+L m+K A(m) \sum_{i=0}^{n-2} a_{i+2} \alpha^{i}\right.\right. \\
& \left.\left.-\sum_{i=1}^{n-2}\left(K a_{i+2} A(m) \alpha^{i}-A^{i}\left(K a_{i+2} A(m)\right)\right)\right) \alpha\right\rfloor \\
& =\left\lfloor\left(M+L m+\sum_{i=0}^{n-2} A^{i}\left(K a_{i+2} A(m)\right)\right) \alpha\right\rfloor .
\end{aligned}
$$


In the first equality we only wrote $m \alpha-A(m)$ for $\{m \alpha\}$. In the second we canceled out the terms $L A(m)$ and $K a_{0} m$, and used $a_{0} \alpha^{-1}=-\sum_{i=0}^{n-1} a_{i+1} \alpha^{i}$ (from (1)). In the third equality we canceled out the term $K a_{1} A(m)$ and took out $\alpha$ as a general multiplier. In the last equality we did more cancellations, and wrote $k=A^{0}(k)$ (for $k=K a_{2} A(m)$ ).

Notes. 1. The identity (2) expresses a compound sum of the iterated functional $A^{i}$ for $0 \leq i \leq n$, operating on an algebraic number of degree $\leq n$, in terms of essentially only $A^{1}(m)$ and $A^{0}(m)=m$. Though the $A^{i}$ appear also in $\theta$, the integer $D=D(m)$ is bounded. In other words, the sum of the $A^{i}$ on the left-hand side of (2) is "almost linear". The free parameters $K, L, M$ permit to shape (2) into various forms, which enables different applications of the formula.

2. We do not require $a_{n} \neq 0$, so $\alpha$ is of degree $\leq n$.

3. Identity (2) holds also for $n=1$ ( $\alpha$ rational).

4. $\theta=0$ for $n<3$.

5. The special case of identity (2) when $n=2$ and $\alpha=\phi=(1+\sqrt{5}) / 2$ (the golden mean) has been proved and used recently in a series of investigations $[19,20,38]$. In [20] the case where $\alpha$ is any algebraic integer of degree 2 was considered.

6. For any real number $\gamma$, define the (nonhomogeneous) Beatty sequence $S(\alpha, \gamma)$ and $A_{\gamma}(m)$ by

$$
S(\alpha, \gamma)=\left\{\lfloor m \alpha+\gamma\rfloor: m \in \mathbb{Z}^{+}\right\}, \quad A_{\gamma}(m)=\lfloor m \alpha+\gamma\rfloor .
$$

It is not hard to see that (2) holds also with $A$ replaced by $A_{\gamma}$, except that (3) has then to be replaced by

$$
D=\left\lfloor M \alpha+\left(L+K a_{0} \alpha^{-1}\right)(\{m \alpha+\gamma\}-\gamma)-\theta \alpha+\gamma\right\rfloor .
$$

If $\alpha$ is as in Theorem 1 and so (2) holds, it may be useful for some applications to have a companion identity for $\beta$, where $\alpha^{-1}+\beta^{-1}=1$. This is done in Corollary 1 below. We first produce in Theorem 2 below a polynomial of degree $\leq n$, which depends on the coefficients of (1), of which $\beta$ is a root.

Theorem 2. Suppose that $\alpha$ is a nonzero root of $f(x)=a_{n} x^{n}+a_{n-1} x^{n-1}+\cdots+a_{0}$ over some field with $\alpha \neq 1$. Let $\beta^{-1}=1-\alpha^{-1}$. Then the polynomial $g(x)=$ $b_{n} x^{n}+b_{n-1} x^{n-1}+\cdots+b_{0}$ has a root $\beta$, where

$$
b_{k}=(-1)^{n-k} \sum_{i=0}^{k}\left(\begin{array}{c}
n-i \\
n-k
\end{array}\right) a_{i} \quad(k=0, \ldots, n) .
$$

Proof. Since $\alpha \neq 0$, the polynomial $a_{n}+\cdots+a_{1} x^{n-1}+a_{0} x^{n}$ has a root $\alpha^{-1}$. Also $a_{n}+\cdots+a_{1}(x+1)^{n-1}+a_{0}(x+1)^{n}$ has a root $\alpha^{-1}-1$. Thus $a_{n}+\cdots+$ $a_{1}(1-x)^{n-1}+a_{0}(1-x)^{n}$ has a root $1-\alpha^{-1}$. If we write

$$
a_{n}+\cdots+a_{1}(1-x)^{n-1}+a_{0}(1-x)^{n}=b_{n}+\cdots+b_{1} x^{n-1}+b_{0} x^{n} \text {, }
$$

then an easy computation shows that

$$
b_{n-k}=(-1)^{k} \sum_{i=0}^{n-k}\left(\begin{array}{c}
n-i \\
k
\end{array}\right) a_{i} \quad(k=0, \ldots, n),
$$


which is equivalent to (4). With these values of $b_{k}$, the polynomial $b_{n}+$ $\cdots+b_{1} x^{n-1}+b_{0} x^{n}$ thus has a root $1-\alpha^{-1}$. Since $\alpha \neq 1$, the polynomial $b_{n} x^{n}+b_{n-1} x^{n-1}+\cdots+b_{0}$ has a root $\beta=1 /\left(1-\alpha^{-1}\right)$.

Write $B(m)=\lfloor m \beta\rfloor$. The following is a companion result to Theorem 1 .

Corollary 1. Under the hypotheses of Theorem 1 and if $\alpha \neq 1$ and $\beta^{-1}=$ $1-\alpha^{-1}$, then

$$
B\left(M+L m+\sum_{i=0}^{n-2} B^{i}\left(K b_{i+2} B(m)\right)\right)=\left(L-K b_{1}\right) B(m)-K b_{0} m+D,
$$

where $D$ is bounded, namely

$$
\begin{aligned}
& D=\left\lfloor M \beta+\left(L+K b_{0} \beta^{-1}\right)\{m \beta\}-\theta \beta\right\rfloor, \\
& \theta=\sum_{i=1}^{n-2}\left(K b_{i+2} B(m) \beta^{i}-B^{i}\left(K b_{i+2} B(m)\right)\right),
\end{aligned}
$$

where $\beta=\left(1-\alpha^{-1}\right)^{-1}$ and $b_{k}$ is given by (4).

\section{DISCRETE CHAOS OR DISCRETE DYNAMICAL SYSTEMS}

D. Hofstadter [26] defined a sequence $\left\{a_{n}\right\}$ by

$$
a_{1}=a_{2}=1, \quad a_{m}=a_{m-a_{m-1}}+a_{m-a_{m-2}} \quad(m \geq 3),
$$

which he called a "strange" recursion, since the subscripts depend on terms of the sequence itself. This and some other "strange" sequences appear to behave quite irregularly and are likely to produce good "pseudo-random" numbers, whereas other "strange" sequences are more regular. The theory of strange recursions has been called "discrete chaos" by Golomb [21]. One of the difficulties of this theory is to decide whether a strange looking sequence is indeed "crazy", or contains regular structure, if hidden. A case in point is the sequence

$$
a_{1}=a_{2}=1, \quad a_{m}=a_{m-a_{m-1}}+a_{a_{m-1}} \quad(m \geq 3)
$$

defined by $\mathrm{J}$. H. Conway, which he believed to have unpredictable convergence behavior, but Mallows [30] established enough of the structure to exhibit the asymptotic behavior.

Rather than discussing discrete chaos in the context of a single sequence, such as the Hofstadter or Conway sequences, we consider here relations involving terms of two sequences $a(m)$ and $b(m)$, which appear in one another indices to arbitrary depth. An example of depth 2 is $a_{b_{m}}-b_{a_{m}}=x(m)$, where $x(m)$ is a suitable function of $m$. Typesetters may be happier to write this in the form $a b(m)-b a(m)=x(m)$. Also mathematicians will be happier with this notation, since it enables to write such relations conveniently to arbitrary depth $k$,

$$
(a b)^{k}(m)-(b a)^{k}(m)=x(m),
$$

where $(a b)^{k}$ and similar expressions in the sequel denote composition. Though in (6) $x$ depends on $k$ in addition to $m$, we wish to investigate (6) for every fixed $k \in \mathbb{Z}^{+}$. 
Call a solution $\{(\{a(m)\},\{b(m)\},\{x(m)\}): m=1,2, \ldots\}$ of $(6)$ chaotic if $x(m)$ is bounded in $m$. If a chaotic solution exists, we also say that (6) is chaotic. Several questions can be asked about relations of the form (6). For example

(i) Find a set $S$ of chaotic solutions for every $k \in \mathbb{Z}^{+}$.

(ii) Find a set $T$ of solutions of (6) for which $x(m)=x$ is constant for all $m$. In this case (6) is a functional equation, and every solution in $T$ is regular (discrete order). For example, any arithmetic progression is in $T$ for (6). Are there additional solutions?

(iii) For every set of chaotic solutions of (6), construct infinite subsequences $\left\{m_{i}\right\} \subseteq \mathbb{Z}$ for which $x=x(m)$ is constant when $m$ is restricted to $\left\{m_{i}\right\}$ in (6).

For approaching these questions, let $t \in \mathbb{Z}^{+}$, and let $\alpha>0$ satisfy

$$
\frac{1}{\alpha}+\frac{1}{\alpha+t}=1
$$

i.e. the polynomial

$$
x^{2}+(t-2) x-t=0
$$

has the positive solution

$$
\alpha=\frac{2-t+\sqrt{t^{2}+4}}{2}
$$

and let

$$
\beta=\alpha+t .
$$

We assume $m \neq 0$ throughout. The special case of (2) for $n=2$ and the polynomial (8) (so $a_{2}=1, a_{1}=t-2, a_{0}=-t$ ) is

$$
\begin{gathered}
A(M+L m+K A(m))=(L-K(t-2)) A(m)+K t m+D, \\
D=\left\lfloor M \alpha+\left(L-K t \alpha^{-1}\right)\{m \alpha\}\right\rfloor .
\end{gathered}
$$

The special case of (5) for $n=2$ and $\beta$ given by (10) (so $\beta$ satisfies $x^{2}-$ $(t+2) x+t=0)$ is

$$
\begin{gathered}
B(M+L m+K B(m))=(L+K(t+2)) B(m)-K t m+D, \\
D=\left\lfloor M \beta+\left(L+K t \beta^{-1}\right)\{m \beta\}\right\rfloor .
\end{gathered}
$$

Define

$$
\delta(m)=\lfloor(\alpha+t-2)\{m \alpha\}\rfloor+1,
$$

which is $-D$ of (11) for $M=L=0, K=1$.

From (9), $1<\alpha<2$, hence

$$
1 \leq \delta(m) \leq t
$$

Put $K=1, L=M=0$ in (11). Then $A^{2}(m)=(2-t) A(m)+t m+$ $\left\lfloor-t \alpha^{-1}\{m \alpha\}\right\rfloor$. By $(8),-t \alpha^{-1}=2-t-\alpha$. Also for $x$ nonintegral, $\lfloor x\rfloor+\lfloor-x\rfloor=$ -1 . Hence

$$
A^{2}(m)=B(m)-(t-1) A(m)-\delta(m) .
$$


Put $K=1, L=M=0$ in (12). Then $B^{2}(m)=(t+2) B(m)-t m+$ $\left\lfloor t \beta^{-1}\{m \beta\}\right\rfloor$. Now $t \beta^{-1}=t+2-\beta=2-\alpha$, so $0<t \beta^{-1}<1$. Hence,

$$
B^{2}(m)=(t+1) B(m)+A(m) .
$$

Put $K=1, L=t, M=0$ in (11). Then

$$
A B(m)=B(m)+A(m) .
$$

Put $K=1, L=-t, M=0$ in (12). Then

$$
B A(m)=B(m)+A(m)-\delta(m) .
$$

Replace $m$ by $B(m)$ in (15). Using (16) and (17), we get

$$
A^{2} B(m)=2 B(m)+(2-t) A(m)-\delta B(m) \text {. }
$$

Replace $m$ by $A(m)$ in (16). Using (18) and (15), we get

$$
B^{2} A(m)=(2+t) B(m)+2 A(m)-(2+t) \delta(m) .
$$

Replace $m$ by $A(m)$ in (17). Using (18) and (15), we get

$$
A B A(m)=2 B(m)+(2-t) A(m)-2 \delta(m) \text {. }
$$

Replace $m$ by $B(m)$ in (18). Using (16) and (17), we get

$$
B A B(m)=(2+t) B(m)+2 A(m)-\delta B(m) \text {. }
$$

These identities help to give a chaotic solution of (6) in Corollary 2 below. First we need a technical result, for which we define, for $t \in \mathbb{Z}$,

$$
\begin{gathered}
g_{k}(t)=2^{k} \sum_{i}\left(\begin{array}{c}
k \\
2 i+1
\end{array}\right)\left(\left(\frac{t}{2}\right)^{2}+1\right)^{i}, \\
f_{k}(t)=\frac{1}{2} g_{k+1}(t)+\frac{t}{2} g_{k}(t), \\
h_{k}(t)=\frac{1}{2} g_{k+1}(t)-\left(1+\frac{t}{2}\right) g_{k}(t) .
\end{gathered}
$$

We remark that since $\left(\begin{array}{l}p \\ q\end{array}\right)=0$ for $q<0$ or $p<q$, a sum such as $\sum_{i}\left(\begin{array}{c}k \\ 2 i+1\end{array}\right)$ is over all $i=0,1, \ldots,\lfloor k / 2\rfloor$. Also note that $g_{k}(t)=g_{k}(-t), \quad g_{0}(t)=0, \quad f_{0}(t)=f_{0}(-t)=1, \quad f_{1}( \pm t)=4 \pm t, \quad h_{0}(t)=1$.

Lemma 1. For $k, t \in \mathbb{Z}^{+}$we have

$$
f_{k}(t)=(2+t) f_{k-1}(t)+2 f_{k-1}(-t),
$$

$$
g_{k}(t)=(2+t) g_{k-1}(t)+2 h_{k-1}(t), \quad h_{k}(t)=2 g_{k-1}(t)+(2-t) h_{k-1}(t) .
$$

Proof. Let $\tau=(t / 2)^{2}+1$. Denote the right-hand side of (26) by $R_{1}$. Using (24) and (23),

$$
\begin{aligned}
R_{1} & =\left(2+\frac{t}{2}\right) g_{k}(t)+\frac{t^{2}}{2} g_{k-1}(t) \\
& =\left(2+\frac{t}{2}\right) 2^{k} \sum_{i}\left(\begin{array}{c}
k \\
2 i+1
\end{array}\right) \tau^{i}+(\tau-1) 2^{k} \sum_{i}\left(\begin{array}{c}
k-1 \\
2 i+1
\end{array}\right) \tau^{i}
\end{aligned}
$$


Let $R=R_{1}-(t / 2) g_{k}(t)$. We use the identity

$$
\left(\begin{array}{l}
l \\
j
\end{array}\right)+\left(\begin{array}{c}
l \\
j+1
\end{array}\right)=\left(\begin{array}{l}
l+1 \\
j+1
\end{array}\right)
$$

Then

$$
\begin{aligned}
R & =2^{k}\left(2 \sum_{i}\left(\begin{array}{c}
k \\
2 i+1
\end{array}\right) \tau^{i}-\sum_{i}\left(\begin{array}{c}
k-1 \\
2 i+1
\end{array}\right) \tau^{i}+\sum_{i}\left(\begin{array}{c}
k-1 \\
2 i+1
\end{array}\right) \tau^{i+1}\right) \\
& =2^{k}\left(\sum_{i}\left(\begin{array}{c}
k \\
2 i+1
\end{array}\right) \tau^{i}+\sum_{i}\left(\begin{array}{c}
k \\
2 i
\end{array}\right) \tau^{i}\right)=2^{k} \sum_{i}\left(\begin{array}{c}
k+1 \\
2 i+1
\end{array}\right) \tau^{i}=\frac{1}{2} g_{k+1}(t),
\end{aligned}
$$

where we used (29) three times. Thus

$$
R_{1}=\frac{1}{2} g_{k+1}(t)+\frac{t}{2} g_{k}(t)=f_{k}(t)
$$

by (24), proving (26). Let $R=\left(f_{k}(-t)+t f_{k-1}(-t)\right) / 2$. By (24) and (23),

$$
\begin{aligned}
R & =\frac{1}{4}\left(g_{k+1}(t)-t^{2} g_{k-1}(t)\right) \\
& =2^{k-1}\left(\sum_{i}\left(\begin{array}{c}
k+1 \\
2 i+1
\end{array}\right) \tau^{i}-(\tau-1) \sum_{i}\left(\begin{array}{c}
k-1 \\
2 i+1
\end{array}\right)\right) .
\end{aligned}
$$

Adding and subtracting $2^{k-1} \sum_{i}\left(\begin{array}{l}k \\ 2 i\end{array}\right) \tau^{i}$ and using (29), we get

$$
R=2^{k} \sum_{i}\left(\begin{array}{c}
k \\
2 i+1
\end{array}\right) \tau^{i}=g_{k}(t)
$$

proving the first part of (27). Now let $R=\left(f_{k}(-t)-t f_{k-1}(-t)\right) / 2$. By (24),

$$
R=\frac{1}{4} g_{k+1}(t)-\frac{t}{2} g_{k}(t)+(\tau-1) g_{k-1}(t) \text {. }
$$

Letting $R_{2}=(\tau-1) g_{k-1}(t)$, we get on using (23),

$$
\begin{aligned}
R_{2} & =(\tau-1) 2^{k-1} \sum_{i}\left(\begin{array}{c}
k-1 \\
2 i+1
\end{array}\right) \tau^{i} \\
& =2^{k-1}\left(\sum_{i}\left(\begin{array}{c}
k-1 \\
2 i-1
\end{array}\right) \tau^{i}-\sum_{i}\left(\begin{array}{c}
k-1 \\
2 i+1
\end{array}\right) \tau^{i}\right) .
\end{aligned}
$$

Adding and subtracting $2^{k-1} \sum_{i}\left(\begin{array}{c}k-1 \\ 2 i\end{array}\right) \tau^{i}$ and using (29) followed by adding and subtracting $2^{k-1} \sum_{i}\left(\begin{array}{c}k \\ 2 i+1\end{array}\right) \tau^{i}$ and using (29), we get

$$
R_{2}=2^{k-1}\left(\sum_{i}\left(\begin{array}{c}
k \\
2 i
\end{array}\right) \tau^{i}-\sum_{i}\left(\begin{array}{c}
k \\
2 i+1
\end{array}\right) \tau^{i}\right)=\frac{1}{4} g_{k+1}(t)-g_{k}(t) \text {. }
$$

Hence

$$
R=\frac{1}{4} g_{k+1}(t)-\frac{t}{2} g_{k}(t)+\frac{1}{4} g_{k+1}(t)-g_{k}(t)=h_{k}(t)
$$

by $(25)$, proving the second part of (27). For proving the first part of (28) we use (25) to write

$$
(2+t) g_{k-1}(t)+2 h_{k-1}(t)=(2+t) g_{k-1}(t)+g_{k}(t)-(2+t) g_{k-1}(t)=g_{k}(t) \text {. }
$$


For proving the second part, we first note directly from the second part of (27) that it holds for $k=1$. Denote the right-hand side of (28) by $R$. For $k>1$ we use (27) (and $g_{k}(t)=g_{k}(-t)$ ) to write

$$
R=\frac{1}{2}\left(2 f_{k-1}(t)-2 t f_{k-2}(t)+(2-t)\left(f_{k-1}(-t)-t f_{k-2}(-t)\right)\right) .
$$

From $(26), f_{k}(-t)=(2-t) f_{k-1}(-t)+2 f_{k-1}(t)$. Hence

$$
R=\frac{1}{2}\left(f_{k}(-t)-t f_{k-1}(-t)\right)=h_{k}(t)
$$

by (27), establishing (28).

Theorem 3. For $\alpha$ and $\beta$ of the form (9) and (10) respectively, $\delta$ given by (13), $k \in \mathbb{Z}^{+}, m \in \mathbb{Z}, m \neq 0$, we have

$$
\begin{array}{r}
(A B)^{k}(m)=f_{k-1}(t) B(m)+f_{k-1}(-t) A(m) \\
-\sum_{i=2}^{k} g_{i-1}(t) \delta B(A B)^{k-i}(m), \\
(B A)^{k}(m)=f_{k-1}(t) B(m)+f_{k-1}(-t) A(m) \\
-\sum_{i=1}^{k} f_{i-1}(t) \delta(B A)^{k-i}(m), \\
A(A B)^{k}(m)=g_{k}(t) B(m)+h_{k}(t) A(m) \\
-\sum_{i=1}^{k} f_{i-1}(-t) \delta B(A B)^{k-i}(m), \\
A(B A)^{k}(m)=g_{k}(t) B(m)+h_{k}(t) A(m) \\
-\sum_{i=1}^{k} g_{i}(t) \delta(B A)^{k-i}(m) .
\end{array}
$$

Proof. We use induction on $k$ for any fixed $m \neq 0$. For $k=1,(30)-(33)$ become (17), (18), (19), (21) respectively. Now suppose (30)-(33) hold for $k$.

Replace $m$ by $A B(m)$ in (30). Then by (22) and (19)

$$
\begin{aligned}
(A B)^{k+1}(m)= & \left((2+t) f_{k-1}(t)+2 f_{k-1}(-t)\right) B(m) \\
& +\left(2 f_{k-1}(t)+(2-t) f_{k-1}(-t)\right) A(m) \\
& -\left(f_{k-1}(t)+f_{k-1}(-t)\right) \delta B(m) \\
& -\sum_{i=2}^{k} g_{i-1}(t) \delta B(A B)^{k+1-i}(m) .
\end{aligned}
$$

Now (24) implies $f_{k-1}(t)+f_{k-1}(-t)=g_{k}(t)$. This and (26) establish the validity of $(30)$.

Replace $m$ by $B A(m)$ in (31). By (20), (21) and (26)

$$
(B A)^{k+1}(m)=f_{k}(t) B(m)+f_{k}(-t) A(m)-\sum_{i=1}^{k+1} f_{i-1}(t) \delta(B A)^{k+1-i}(m),
$$


establishing (31). Now replace $m$ by $A B(m)$ in (32). Using (22), (19) and (28),

$$
\begin{aligned}
A(A B)^{k+1}(m)= & g_{k+1}(t) B(m)+h_{k+1}(t) A(m) \\
& -\sum_{i=1}^{k+1} f_{i-1}(-t) \delta B(A B)^{k+1-i}(m),
\end{aligned}
$$

where we used $g_{k}(t)+h_{k}(t)=f_{k}(-t)$ (by (27)). Finally, replace $m$ by $B A(m)$ in (33). Using (20), (21) and (28)

$$
A(B A)^{k+1}(m)=g_{k+1}(t) B(m)+h_{k+1}(t) A(m)-\sum_{i=1}^{k+1} g_{i}(t) \delta(B A)^{k+1-i}(m),
$$

establishing the validity of (33).

Corollary 2. For $k \in \mathbb{Z}^{+}, m \in \mathbb{Z}, m \neq 0$,

$$
\begin{aligned}
& (A B)^{k}(m)-(B A)^{k}(m) \\
& =\sum_{i=1}^{k} f_{i-1}(t) \delta(B A)^{k-i}(m)-\sum_{i=2}^{k} g_{i-1}(t) \delta B(A B)^{k-i}(m), \\
& A(A B)^{k}(m)-A(B A)^{k}(m) \\
& =\sum_{i=1}^{k}\left(g_{i}(t) \delta(B A)^{k-i}(m)-f_{i-1}(-t) \delta B(A B)^{k-i}(m)\right) .
\end{aligned}
$$

Proof. Follows directly from Theorem 3.

It follows from (14) that the right-hand sides of (34) and (35) are bounded in $m$, so (34) provides a chaotic solution for (6), and (35) a chaotic solution for a similar relation. Many similar chaotic relations involving $A, B$ can be established. We remark that any finite word over the alphabet $\{A, B\}$ can be expressed uniquely in terms of $B(m), A(m)$ and $\delta(A, B)(m)$, where $\delta(A, B)(m)$ denotes any finite sum of $\delta$ operating on words over $\{A, B\}$. It can also be expressed uniquely in terms of $B(m), m$ and $\delta(A, B)(m)$ and also in terms of $A(m), m$ and $\delta(A, B)$. The latter case, for the special instance $\alpha=\phi$ $(t=1)$, for which $\delta(m)=1$ for all $m$, was shown in [8].

For the special case $k=1$ of (34) we can say a little more than being chaotic. A function $Q: \mathbb{Z}^{+} \rightarrow \mathbb{Z}^{+}$is periodic if there are $m_{0}, p \in \mathbb{Z}^{+}$such that $Q(m+p)=Q(m)$ for all $m \geq m_{0}$. Otherwise it is aperiodic.

Theorem 4. For $k=1$, identity (34) is aperiodic for every $t \geq 2$, and every integer in $[1, t]$ is assumed infinitely often.

Proof. For $k=1$, (34) has the form $A B(m)-B A(m)=\delta(m)$. Let $\zeta=$ $\alpha+t-2$. Then $t-1<\zeta<t$, and $\delta(m)=\lfloor\{m \alpha\} \zeta\rfloor+1$. Suppose there exist $m_{0}, p \in \mathbb{Z}^{+}$such that $\delta(m+p)=\delta(m)$ for all $m \geq m_{0}$. Then also $\delta(m)=\delta(m+l p)$ for all $m \geq m_{0}$ and all $l \in \mathbb{Z}^{+}$. Choose some $m_{1} \geq m_{0}$ such that $\left\lfloor\left\{m_{1} \alpha\right\} \zeta\right\rfloor=0$. This can be done in view of the density of $\{m \alpha\}$. Then also $\left\lfloor\left\{\left(m_{1}+l p\right) \alpha\right\} \zeta\right\rfloor=\left\lfloor\left\{m_{1} \alpha+l(p \alpha)\right\} \zeta\right\rfloor=0$ for all $l=\mathbb{Z}^{+}$. But since $p \alpha$ is irrational, $\left\{m_{1} \alpha+l(p \alpha)\right\}$ can be made arbitrarily close to 1 , so $\left\lfloor\left\{\left(m_{1}+l p\right) \alpha\right\} \zeta\right\rfloor=t-1 \geq 1$, a contradiction. The last part follows from the density of $\{m \alpha\}$ and (13), (14). 


\section{DISCRETE ORDER}

Theorem 3 enables to exhibit certain regular, nonchaotic behavior for the special case $t=1$. As usual we define the Fibonacci numbers $F_{i}$ by

$$
F_{-1}=0, \quad F_{0}=1, \quad F_{k}=F_{k-1}+F_{k-2} \quad(k \geq 1) .
$$

Theorem 5. For $t=1$, i.e., $\alpha=\phi=(1+\sqrt{5}) / 2$ and $\beta=\alpha+1, k \in \mathbb{Z}^{+}$, $m \in \mathbb{Z}, m \neq 0$, we have

$$
\begin{aligned}
(A B)^{k}(m) & =F_{3 k-2} B(m)+F_{3 k-3} A(m)-\frac{1}{2}\left(F_{3 k-2}-1\right), \\
(B A)^{k}(m) & =F_{3 k-2} B(m)+F_{3 k-3} A(m)-\frac{1}{2}\left(F_{3 k}-1\right), \\
A(A B)^{k}(m) & =F_{3 k-1} B(m)+F_{3 k-2} A(m)-\frac{1}{2} F_{3 k-1}, \\
A(B A)^{k}(m) & =F_{3 k-1} B(m)+F_{3 k-2} A(m)-\frac{1}{2}\left(F_{3 k+1}-1\right) .
\end{aligned}
$$

Proof. Since by (14) $\delta(m)=1$ for all $m \in \mathbb{Z}$ when $t=1$, it suffices to show, in view of Theorem 3 ,

$$
\begin{array}{cl}
f_{k-1}(1)=F_{3 k-2}, \quad f_{k-1}(-1)=F_{3 k-3}, & g_{k}(1)=F_{3 k-1}, \quad h_{k}(1)=F_{3 k-2}, \\
\text { (40) } \quad \sum_{i=2}^{k} g_{i-1}(1)=\frac{1}{2}\left(F_{3 k-2}-1\right), & \sum_{i=1}^{k} f_{i-1}(1)=\frac{1}{2}\left(F_{3 k}-1\right), \\
& \sum_{i=1}^{k} f_{i-1}(-1)=\frac{1}{2} F_{3 k-1}, \quad \sum_{i=1}^{k} g_{i}(1)=\frac{1}{2}\left(F_{3 k+1}-1\right) .
\end{array}
$$

The following identity is well known:

$$
F_{k}=\frac{1}{\sqrt{5}}\left(\left(\frac{1+\sqrt{5}}{2}\right)^{k+1}-\left(\frac{1-\sqrt{5}}{2}\right)^{k+1}\right) .
$$

Now $((1 \pm \sqrt{5}) / 2)^{3}=2 \pm \sqrt{5}$. Hence

$$
F_{3 k-1}=\frac{1}{\sqrt{5}}\left((2+\sqrt{5})^{k}-(2-\sqrt{5})^{k}\right) .
$$

Thus,

$$
\begin{aligned}
F_{3 k-1} & =\frac{1}{\sqrt{5}}\left(\sum_{i}\left(\begin{array}{c}
k \\
i
\end{array}\right) 5^{i / 2} 2^{k-i}-\sum_{i}\left(\begin{array}{c}
k \\
i
\end{array}\right)(-1)^{i} 5^{i / 2} 2^{k-i}\right) \\
& =\sum_{i}\left(\begin{array}{c}
k \\
2 i+1
\end{array}\right) 5^{i} 2^{k-2 i} .
\end{aligned}
$$

Hence by (23),

$$
g_{k}(1)=F_{3 k-1}
$$

Now

$$
\left(\frac{1 \pm \sqrt{5}}{2}\right)^{3 k-1}=\left(\left(\frac{1 \pm \sqrt{5}}{2}\right)^{3}\right)^{k} \frac{2}{1 \pm \sqrt{5}}=(2 \pm \sqrt{5})^{k}\left(\frac{-1 \pm \sqrt{5}}{2}\right)
$$


Hence by (25) and (29),

$$
\begin{aligned}
F_{3 k-2} & =\frac{1}{\sqrt{5}}\left(\left(\frac{1+\sqrt{5}}{2}\right)^{3 k-1}-\left(\frac{1-\sqrt{5}}{2}\right)^{3 k-1}\right) \\
& =\frac{1}{\sqrt{5}}\left(\frac{\sqrt{5}-1}{2} \sum_{i}\left(\begin{array}{c}
k \\
i
\end{array}\right) 5^{i / 2} 2^{k-i}+\frac{\sqrt{5}+1}{2} \sum_{i}\left(\begin{array}{c}
k \\
i
\end{array}\right)(-1)^{i} 5^{i / 2} 2^{k-i}\right) \\
& =\sum_{i}\left(\begin{array}{c}
k \\
2 i
\end{array}\right) 5^{i} 2^{k-2 i}-\sum_{i}\left(\begin{array}{c}
k \\
2 i+1
\end{array}\right) 5^{i} 2^{k-2 i-1}=h_{k}(1) .
\end{aligned}
$$

From (26), $f_{k}(-1)=f_{k-1}(-1)+2 f_{k-1}(1)$, so $h_{k}(1)=f_{k-1}(1)$ by (27). Thus,

$$
f_{k-1}(1)=h_{k}(1)=F_{3 k-2} \text {. }
$$

Again from (26), $2 f_{k-1}(-1)=f_{k}(1)-3 f_{k-1}(1)=F_{3 k+1}-3 F_{3 k-2}$, so

$$
f_{k-1}(-1)=F_{3 k-3} \text {. }
$$

Identities (40) and (41) are seen to hold for $k=1$. Suppose they hold for $k$. Then by (43), $\sum_{i=2}^{k+1} g_{i-1}(1)=\left(F_{3 k-2}+1\right) / 2+F_{3 k-1}=\left(F_{3 k+1}-1\right) / 2$. By (44), $\sum_{i=1}^{k+1} f_{i-1}(1)=\left(F_{3 k}-1\right) / 2+F_{3 k+1}=\left(F_{3 k+3}-1\right) / 2$, establishing (40). By (45), $\sum_{i=1}^{k+1} f_{i-1}(-1)=F_{3 k-1} / 2+F_{3 k}=F_{3 k+2} / 2$. Finally by (43), $\sum_{i=1}^{k+1} g_{i}(1)=\left(F_{3 k+1}-1\right) / 2+F_{3 k+2}=\left(F_{3 k+4}-1\right) / 2$, establishing $(41)$.

Corollary 3. Under the hypotheses of Theorem 5 ,

$$
\begin{aligned}
(A B)^{k}(m)-(B A)^{k}(m) & =\frac{1}{2} F_{3 k-1}, \\
A(A B)^{k}(m)-A(B A)^{k}(m) & =\frac{1}{2}\left(F_{3 k}-1\right) .
\end{aligned}
$$

Proof. Follows immediately from Theorem 5.

Note that by equality (42), the right-hand side of (46) has the form $\frac{1}{2 \sqrt{5}}\left((2+\sqrt{5})^{k}-(2-\sqrt{5})^{k}\right)$, which is also the solution of the recursion

$$
C_{0}=0, \quad C_{1}=1, \quad C_{k}=4 C_{k-1}+C_{k-2} \quad(k \geq 2),
$$

which has been considered in [47]. See [45]. Formulas of the form (46) and (47) have also been derived in [8] from the theory of Fibonacci numbers. Another identity of this form is $B(A B)^{k}(m)-B(B A)^{k}(m)=\frac{1}{2}\left(F_{3 k}+1\right)$.

\section{CONSTRUCTION OF INFINITE NONCHAOTIC SUBSEQUENCES OF CHAOTIC SEQUENCES}

We shall illustrate the construction with the special case $k=2$ of (34), namely,

$$
(A B)^{2}(m)-(B A)^{2}(m)=(4+t) \delta(m)-2 \delta B(m)+\delta B A(m) .
$$

We begin by collecting some relevant facts from the theory of continued fractions. See e.g. $[25,37,34]$. The simple continued fraction of a real number $\alpha$ is

$$
\alpha=c_{0}+\frac{1}{c_{1}+\frac{1}{c_{2}+\frac{1}{\ddots}}}=\left[c_{0}, c_{1}, c_{2}, \ldots\right],
$$


where the $c_{i}$ are positive integers for $i \geq 1, c_{0}$ any integer. The convergents of $\alpha$ are the numbers $p_{i} / q_{i}=\left[c_{0}, \ldots, c_{i}\right]$, and they satisfy

$$
\begin{aligned}
& p_{-1}=1, \quad p_{0}=c_{0}, \quad p_{m}=c_{m} p_{m-1}+p_{m-2} \quad(m \geq 1), \\
& q_{-1}=0, \quad q_{0}=1, \quad q_{m}=c_{m} q_{m-1}+q_{m-2} \quad(m \geq 1) .
\end{aligned}
$$

We remark that these recursions are valid for any real numbers $c_{0}, c_{1}, \ldots$.

Every real irrational number $\alpha$ has a unique representation as an infinite simple continued fraction. We also define

$$
c_{m}^{\prime}=\left[c_{m}, c_{m+1}, \ldots\right]=c_{m}+\frac{1}{c_{m+1}^{\prime}} .
$$

Then

$$
\begin{aligned}
\alpha & =c_{0}^{\prime}=\left[c_{0}, c_{1}^{\prime}\right]=\frac{c_{0} c_{1}^{\prime}+1}{c_{1}^{\prime}}=\left[c_{0}, \ldots, c_{m-1}, c_{m}^{\prime}\right] \\
& =\frac{c_{m}^{\prime} p_{m-1}+p_{m-2}}{c_{m}^{\prime} q_{m-1}+q_{m-2}}=\frac{p_{m}^{\prime}}{q_{m}^{\prime}},
\end{aligned}
$$

where $p_{m}^{\prime}=c_{m}^{\prime} p_{m-1}+p_{m-2}$ and $q_{m}^{\prime}=c_{m}^{\prime} q_{m-1}+q_{m-2}$. The following identities hold:

$$
p_{m} q_{m-1}-p_{m-1} q_{m}=(-1)^{m-1}, \quad p_{m} q_{m-2}-p_{m-2} q_{m}=(-1)^{m} c_{m},
$$

hence, in particular, $p_{m} / q_{m}$ is in lowest terms. Now (50) and (51) imply directly, $q_{m} \alpha-p_{m}=(-1)^{m} / q_{m+1}^{\prime}$. Thus,

$$
q_{2 m} \alpha-p_{2 m}=\frac{1}{q_{2 m+1}^{\prime}}, \quad q_{2 m+1} \alpha-p_{2 m+1}=-\frac{1}{q_{2 m+2}^{\prime}} \quad(m \geq 0) .
$$

In view of (48), we wish to compute $\delta, \delta B, \delta B A$ for the subsequence of the denominators of the convergents. For doing this we need the following three propositions, which relate to numeration systems induced by the convergents $p_{i} / q_{i}$ of a simple continued fraction $\alpha=\left[c_{0}, c_{1}, \ldots\right], \alpha$ irrational.

Proposition II. Every nonnegative integer $N$ has precisely one representation of the form

$$
N=\sum_{i=0}^{k} r_{i} p_{i}, \quad 0 \leq r_{i} \leq c_{i+1} ; \quad r_{i+1}=c_{i+2} \Rightarrow r_{i}=0 \quad(i \geq 0),
$$

and also precisely one representation of the form

$$
N=\sum_{i=0}^{l} s_{i} q_{i}, \quad 0 \leq s_{0}<c_{1}, \quad 0 \leq s_{i} \leq c_{i+1} ; \quad s_{i}=c_{i+1} \Rightarrow s_{i-1}=0 \quad(i \geq 1) .
$$

See [16].

Proposition III. The following identities hold:

$$
\begin{aligned}
p_{2 m+1}-1 & =c_{2 m+1} p_{2 m}+c_{2 m-1} p_{2 m-2}+\cdots+c_{1} p_{0}, \\
p_{2 m}-1 & =c_{2 m} p_{2 m-1}+c_{2 m-2} p_{2 m-3}+\cdots+c_{2} p_{1}, \\
q_{2 m+1}-1 & =c_{2 m+1} q_{2 m}+c_{2 m-1} q_{2 m-2}+\cdots+c_{3} q_{2}+\left(c_{1}-1\right) q_{0}, \\
q_{2 m}-1 & =c_{2 m} q_{2 m-1}+c_{2 m-2} q_{2 m-3}+\cdots+c_{2} p_{1} .
\end{aligned}
$$


Proof. We prove e.g. the penultimate identity. Using (49), the right-hand side $=\left(q_{2 m+1}-q_{2 m-1}\right)+\left(q_{2 m-1}-q_{2 m-3}\right)+\cdots+\left(q_{1}-1\right)=q_{2 m+1}-1$.

Proposition IV. For $0 \leq s_{0}<c_{1}, 0 \leq s_{i} \leq c_{i+1}$ and $s_{i}=c_{i+1} \Rightarrow s_{i-1}=0$ $(i \geq 1)$, we have

$$
\begin{gathered}
N=\sum_{i=2 k}^{l} s_{i} q_{i} \Rightarrow\lfloor N \alpha\rfloor=\sum_{i=2 k}^{l} s_{i} p_{i} \quad\left(s_{2 k} \neq 0, \quad k \geq 0\right), \\
N=\sum_{i=2 k+1}^{l} s_{i} q_{i} \Rightarrow\lfloor N \alpha\rfloor=-1+\sum_{i=2 k+1}^{l} s_{i} p_{i} \\
=\sum_{i=2 k+2}^{l} s_{i} p_{i}+\left(s_{2 k+1}-1\right) p_{2 k+1} \\
\quad+\sum_{i=0}^{k} c_{2 i+1} p_{2 i} \quad\left(s_{2 k+1} \neq 0, \quad k \geq 0\right) .
\end{gathered}
$$

For a proof see [17]. (The second equality of (54) follows from Proposition III.)

For $\alpha$ of the form (9) we have $\alpha=[1, t, t, \ldots]$, so we will apply the above results for the special case $a_{0}=1, a_{i}=t(i \geq 1)$. For example, (49) becomes

$$
\begin{aligned}
& p_{-1}=1, \quad p_{0}=1, \quad p_{m}=t p_{m-1}+p_{m-2} \quad(m \geq 1), \\
& q_{-1}=0, \quad q_{0}=1, \quad q_{m}=t q_{m-1}+q_{m-2} \quad(m \geq 1) .
\end{aligned}
$$

Lemma 2. For $\alpha$ of the form (9) and for every $t \in \mathbb{Z}^{+}$we have $p_{m}=q_{m}+q_{m-1}$ $(m \geq 0)$.

Proof. The recursions (55) imply the assertion for $m=0$. If it holds for $m$, then

$$
p_{m+1}=t p_{m}+p_{m-1}=t\left(q_{m}+q_{m-1}\right)+q_{m-1}+q_{m-2}=q_{m+1}+q_{m} .
$$

To simplify the arguments below we assume $t>1$ throughout, since the case $t=1$ is anyway subsumed by Corollary 3 above.

Theorem 6. There is an integer $m_{0}=m_{0}(t)$ such that for all $m \geq m_{0}$ and $t \geq 2$,

$$
\begin{aligned}
& (A B)^{2}\left(q_{2 m}\right)-(B A)^{2}\left(q_{2 m}\right)=2 t+1, \\
& (A B)^{2}\left(q_{2 m+1}\right)-(B A)^{2}\left(q_{2 m+1}\right)= \begin{cases}t^{2}+3 t+1 & \text { if } t=2 \text { or } 3, \\
t^{2}+3 t & \text { if } t \geq 4,\end{cases}
\end{aligned}
$$

where $p_{i} / q_{i}$ is the ith convergent of $\alpha$.

Proof. By (53), $\left\lfloor q_{2 m} \alpha\right\rfloor=p_{2 m}$, so by (52),

$$
\left\{q_{2 m} \alpha\right\}=q_{2 m} \alpha-\left\lfloor q_{2 m} \alpha\right\rfloor=q_{2 m} \alpha-p_{2 m}=\frac{1}{q_{2 m+1}^{\prime}} \rightarrow 0
$$

as $m \rightarrow \infty$. Hence by (13), for large $m$,

$$
\delta\left(q_{2 m}\right)=1 \text {. }
$$


By (53), Lemma 2 and (55),

$$
\begin{aligned}
B\left(q_{2 m}\right) & =A\left(q_{2 m}\right)+t q_{2 m}=p_{2 m}+t q_{2 m} \\
& =q_{2 m}+q_{2 m-1}+t q_{2 m}=q_{2 m+1}+q_{2 m} .
\end{aligned}
$$

Thus by (53), $\left\lfloor B\left(q_{2 m}\right) \alpha\right\rfloor=\left\lfloor\left(q_{2 m+1}+q_{2 m}\right) \alpha\right\rfloor=p_{2 m+1}+p_{2 m}$, so by (52),

$$
\left\{B\left(q_{2 m}\right) \alpha\right\}=\left(q_{2 m+1}+q_{2 m}\right) \alpha-\left(p_{2 m+1}+p_{2 m}\right)=-\frac{1}{q_{2 m+2}^{\prime}}+\frac{1}{q_{2 m+1}^{\prime}} \rightarrow 0
$$

as $m \rightarrow \infty$. Hence for large $m$,

$$
\delta B\left(q_{2 m}\right)=1 .
$$

From (18), (53) and Lemma 2 we have for large $m$,

$$
\begin{aligned}
B A\left(q_{2 m}\right) & =2 A\left(q_{2 m}\right)+t q_{2 m}-\delta\left(q_{2 m}\right)=2 p_{2 m}+t q_{2 m}-1 \\
& =2 q_{2 m}+2 q_{2 m-1}+t q_{2 m}-1=q_{2 m+1}+2 q_{2 m}+q_{2 m-1}-1 .
\end{aligned}
$$

Hence by Proposition III,

$$
B A\left(q_{2 m}\right)=q_{2 m+1}+2 q_{2 m}+t\left(q_{2 m-2}+q_{2 m-4}+\cdots+q_{2}\right)+(t-1) q_{0} .
$$

This has the form required by Proposition IV if $t>2$. Thus by (53) we have for $t>2$ and large $m$,

$$
\begin{aligned}
\left\lfloor B A\left(q_{2 m}\right) \alpha\right\rfloor & =p_{2 m+1}+2 p_{2 m}+t\left(p_{2 m-2}+p_{2 m-4}+\cdots+p_{2}\right)+(t-1) p_{0} \\
& =p_{2 m+1}+2 p_{2 m}+p_{2 m-1}-1-p_{0} .
\end{aligned}
$$

Hence for $t>2$ and large $m$,

$$
\left\{B A\left(q_{2 m}\right) \alpha\right\}=\left(q_{2 m+1}+2 q_{2 m}+q_{2 m-1}-1\right) \alpha-\left(p_{2 m+1}-2 p_{2 m}+p_{2 m-1}-2\right)
$$

$\rightarrow 2-\alpha$ as $m \rightarrow \infty$. For $t=2$ we have $B A(2 m)=q_{2 m+1}+\left(q_{2 m+1}-1\right)$, which leads to the same asymptotic value $2-\alpha$ for $\left\{B A\left(q_{2 m}\right) \alpha\right\}$. By (13) and (8) we thus have for large $m$,

$$
\delta B A\left(q_{2 m}\right)=\lfloor(\alpha+t-2)(2-\alpha)\rfloor+1=t-3+\lfloor 2 \alpha\rfloor=t-1 \quad(t \geq 2) .
$$

By (56), (57), (58) we thus have for large $m$,

$$
(4+t) \delta(m)-2 \delta B(m)+\delta B A(m)=2 t+1 .
$$

The first asymptotic identity of the theorem follows now from (48).

By (54), $\left\lfloor q_{2 m+1} \alpha\right\rfloor=p_{2 m+1}-1$, hence $\left\{q_{2 m+1} \alpha\right\}=q_{2 m+1} \alpha-p_{2 m+1}+1 \rightarrow 1$ as $m \rightarrow \infty$, hence for large $m$,

$$
\delta\left(q_{2 m+1}\right)=t .
$$

By (54), Proposition III and Lemma 2,

$$
\begin{aligned}
B\left(q_{2 m+1}\right) & =A\left(q_{2 m+1}\right)+t q_{2 m+1}=p_{2 m+1}-1+t q_{2 m+1} \\
& =q_{2 m+1}+q_{2 m}+t q_{2 m+1}-1=q_{2 m+2}+q_{2 m+1}-1 \\
& =q_{2 m+2}+t\left(q_{2 m}+q_{2 m-2}+\cdots+q_{2}\right)+(t-1) q_{0} .
\end{aligned}
$$

Therefore by (53) and Proposition III,

$$
\begin{aligned}
\left\lfloor B\left(q_{2 m+1}\right) \alpha\right\rfloor & =p_{2 m+2}+t\left(p_{2 m}+p_{2 m-2}+\cdots+p_{2}\right)+(t-1) p_{0} \\
& =p_{2 m+2}+p_{2 m+1}-2 .
\end{aligned}
$$


Hence

$\left\{B\left(q_{2 m+1}\right) \alpha\right\}=\left(q_{2 m+2}+q_{2 m+1}-1\right) \alpha-\left(p_{2 m+2}+p_{2 m-1}-2\right) \rightarrow 2-\alpha \quad$ as $m \rightarrow \infty$. As for $\delta B A\left(q_{2 m}\right)$ we thus get for large $m$,

$$
\delta B\left(q_{2 m+1}\right)=t-1 \quad(t \geq 2) .
$$

From (18), (54) and Lemma 2 we get for large $m$,

$$
\begin{aligned}
& B A\left(q_{2 m+1}\right)=2 A\left(q_{2 m+1}\right)+t q_{2 m+1}-\delta\left(q_{2 m+1}\right)=2\left(p_{2 m+1}-1\right)+t q_{2 m+1}-t \\
& \quad=2 q_{2 m+1}+2 q_{2 m}+t q_{2 m+1}-t-2=q_{2 m+2}+2 q_{2 m+1}+\left(q_{2 m}-1\right)-t-1 \\
& =q_{2 m+2}+2 q_{2 m+1}+t\left(q_{2 m-1}+q_{2 m-3}+\cdots+q_{3}\right)+(t-2) q_{1}+(t-1) q_{0} .
\end{aligned}
$$

Thus for $t>2$, (53) implies for large $m$,

$$
\begin{gathered}
\left\lfloor B A\left(q_{2 m+1}\right) \alpha\right\rfloor=p_{2 m+2}+2 p_{2 m+1}+t\left(p_{2 m-1}+p_{2 m-3}+\cdots+p_{3}\right)+(t-2) p_{1} \\
+(t-1) p_{0}=p_{2 m+2}+2 p_{2 m+1}+p_{2 m}-2 p_{1}+t-2 .
\end{gathered}
$$

Therefore for $t>2$ and large $m$,

$\left\{B A\left(q_{2 m+1}\right) \alpha\right\}=\left(q_{2 m+2}+2 q_{2 m+1}+q_{2 m}-t-2\right) \alpha-\left(p_{2 m+2}+2 p_{2 m+1}+p_{2 m}-t-4\right)$

$\rightarrow t+4-(t+2) \alpha$ as $m \rightarrow \infty$. Thus for large $m$,

$$
\delta B A\left(q_{2 m+1}\right)=\lfloor(\alpha+t-2)(t+4-(t-12) \alpha)\rfloor+1=\lfloor(t+4) \alpha\rfloor-7 .
$$

Using the Binomial Theorem we get

$$
\delta B A\left(q_{2 m+1}\right)= \begin{cases}t-1 & \text { for } t=2,3 \\ t-2 & \text { for } t \geq 4 .\end{cases}
$$

Hence by (59), (60), (61), we have for large $m$,

$$
(4+t) \delta(m)-2 \delta B(m)+\delta B A(m)=t^{2}+3 t+1-\varepsilon,
$$

where $\varepsilon=0$ for $t=2,3$ and $\varepsilon=1$ for $t \geq 4$.

\section{BeATTY SUbSEQUENCES}

Recall the notation $S(\alpha, \gamma)=\{\lfloor m \alpha+\gamma\rfloor: m=1,2, \ldots\}$ for a Beatty sequence. By a subsequence we shall mean a proper subsequence throughout. A trivial Beatty subsequence of $S(\alpha, \gamma)$ is $S(l \alpha, \gamma)$ for every integer $l>1$. A Beatty subsequence $S(\zeta, \eta) \subset S(\alpha, \gamma)$ is nontrivial if $\zeta / \alpha$ is irrational. Are there nontrivial Beatty subsequences?

Proposition V (Graham [1973]). Every irrational DCS is a convolution of an irrational DCS $\{S(\alpha, \gamma), S(\beta, \delta)\}$ with two integer DCS, i.e., it has the form

$$
\left\{\bigcup_{i=1}^{r} S\left(a_{i} \alpha, b_{i} \alpha+\gamma\right)\right\} \cup\left\{\bigcup_{i=1}^{r^{\prime}} S\left(a_{i}^{\prime} \beta, b_{i}^{\prime} \beta+\delta\right)\right\} \text {, }
$$

where $\left\{S\left(a_{i}, b_{i}\right): 1 \leq i \leq r\right\}$ and $\left\{S\left(a_{i}^{\prime}, b_{i}^{\prime}\right): 1 \leq i \leq r^{\prime}\right\}$ are two integer DCS.

In a way, Proposition V says that every irrational DCS with more than two moduli is trivial, in the sense that the moduli are integer multiples of two basic "kernel" moduli. Only the ratio of the two kernel moduli is irrational. Does Proposition V imply that there are no nontrivial Beatty subsequences? For answering this question and another one stated below, it is useful to prove the following result. 
Theorem 7. For $\alpha$ and $\beta$ of the form (9) and (10) respectively and for every fixed $k \in\{1,2, \ldots, t\}$ we have

$$
k B(m)+A(m)+l \in S(\alpha, 0) \text { for all } l \in\{0,1, \ldots, k\} .
$$

Proof. Put $K=k, L=k(t-1)+1, M=l$ in (11). Then

$$
\begin{gathered}
A(l+k(t-1) m+m+k A(m))=(k+1) A(m)+k t m+D \\
D=\lfloor l \alpha+(k(t-1)+1-k(\alpha+t-2))\{m \alpha\}\rfloor=\lfloor l \alpha+(1-k(\alpha-1))\{m \alpha\}\rfloor .
\end{gathered}
$$

Now

$$
k(\alpha-1)=\frac{k}{t+\frac{1}{t+\frac{1}{t+}}}<1,
$$

for $k \leq t$. Hence $1-k(\alpha-1)>0$, so $l \alpha+(1-k(\alpha-1))\{m \alpha\}>l \alpha>l$. Also

$$
\begin{aligned}
& l \alpha+(1-k(\alpha-1))\{m \alpha\} \\
& \quad<l \alpha+1-k(\alpha-1)=l+1-(k-l)(\alpha-1) \leq l+1,
\end{aligned}
$$

for $l \leq k$. Hence $D=l$, so

$$
A(k B(m)-(k-1) m+l)=k B(m)+A(m)+l \quad(l=0,1, \ldots, k),
$$

which implies (62).

Corollary 4. For $\alpha$ and $\beta$ of the form (9) and (10) respectively, $S(\alpha+k \beta, 0)$ is a nontrivial Beatty subsequence of $S(\alpha, 0)$ for every $k \in\{1, \ldots, t\}$.

Proof. The nontriviality is trivial, so to speak, since $(\alpha+k \beta) / \alpha=k \beta+1-$ $k$, which is clearly irrational. The $m$ th term of $S(\alpha+k \beta, 0)$ has the form $C_{k}(m)=\lfloor m(\alpha+k \beta)\rfloor$. Now

$$
\begin{aligned}
A(m)+k B(m) & \leq\lfloor m \alpha\rfloor+\lfloor k m \beta\rfloor \leq C_{k}(m) \\
& \leq m \alpha+k m \beta<A(m)+k B(m)+k+1 .
\end{aligned}
$$

Thus

$$
k B(m)+A(m) \leq C_{k}(m) \leq k B(m)+A(m)+k .
$$

The result now follows since by Theorem 7 every integer in the interval $[k B(m)$ $+A(m), k B(m)+A(m)+k]$ is in $S(\alpha, 0)$.

Since by (7) $\alpha^{-1}+\beta^{-1}=1$, Proposition I implies that $\{S(\alpha, 0), S(\beta, 0)\}$ is a DCS for $\alpha, \beta$ of the form (9), (10). Now $t+1<\beta=\alpha+t<t+2$, so consecutive terms of $S(\beta, 0)$ are at distances $\lfloor\beta\rfloor=t+1$ or $\lceil\beta\rceil=t+2$ from one another, where $\lceil x\rceil$ is the smallest integer $\geq x$. It follows that $S(\alpha, 0)$ consists of short blocks of $t$ consecutive integers of long blocks of $t+1$ consecutive integers, separated by a single-integer gap. What is the precise location of all the long blocks within $S(\alpha, 0)$ ? 
Corollary 5. For $\alpha, \beta$ of the form (9), (10), the long blocks in $S(\alpha, 0)$ are precisely the intervals

$$
\begin{aligned}
{[A(t B(m)-} & (t-1) m)=t B(m)+A(m), \\
& A(t B(m)-(t-1) m+t)=t B(m)+A(m)+t] .
\end{aligned}
$$

Proof. From (63) with $k=t$, it follows that $A(t B(m)-(t-1) m)$ is the beginning of a long block for every $m$. We have to show that these are the beginnings of all the long blocks. Again by (63), the gap between two consecutive long block beginnings as given by this formula is $G=t(B(m+1)-B(m))+A(m-1)-A(m)$. Now $A(m+1)-A(m) \in\{1,2\}$, and $B(m+1)-B(m) \in\{t+1, t+2\}$. Moreover, since $\beta=\alpha+t$, it follows that $A(m+1)-A(m)=1$ if and only if $B(m+1)-B(m)=t+1$. Thus $G=t(t+1)+1$ or $G=t(t+2)+2$.

It follows from [7], that any two consecutive long blocks in $S(\alpha, 0)$ are separated either by $t$ or by $t-1$ short blocks. Every block is followed by a one-integer gap. Hence the gap between two consecutive long block beginnings is $G_{1}=t+2+(t-1)(t+1)=t(t+1)+1$, or $G_{1}=t+2+t(t+1)=t(t+2)+2$. The result now follows since $G$ and $G_{1}$ have the same two possible values.

It follows from [43], that if there exist positive integers $a, b$ and positive irrational numbers $\zeta, \eta$, such that $a \zeta^{-1}+b \eta^{-1}=1$, then $S(\zeta, 0)$ and $S(\eta, 0)$ are disjoint. Since by $(7), \alpha^{-1}+\beta^{-1}=1$, it follows from Proposition I that every subsequence of $S(\alpha, 0)$ is disjoint from $S(\beta, 0)$. It is easily seen that

$$
\frac{k+1}{\beta}+\frac{1+k t-k^{2}}{k \beta+\alpha}=1, \quad 1+k t-k^{2} \geq 1 \text { for } 0 \leq k \leq t .
$$

Hence Corollary 4 can also be deduced from Skolem's result. However, Theorem 7 and Corollary 5 do not seem to follow from this proof. Writing $\lfloor m(k \beta+\alpha)\rfloor=\lfloor x \alpha\rfloor$ leads to $\lfloor x \alpha\rfloor=k B(m)+A(m)+l$ for some $l \in\{0, \ldots, k\}$ rather than for all $l \in\{0, \ldots, k\}$, as needed for Corollary 5 .

We now ask a related question. If $S(\zeta, \tau) \subset S(\alpha, 0)$, a Beatty sequence of the form $S(\eta, w)$ is a complement of $S(\zeta, \tau)$ (with respect to $S(\alpha, 0)$ ), if $S(\zeta, \tau) \cup S(\eta, w)=S(\alpha, 0)$ and $S(\zeta, \tau) \cap S(\eta, w)=\varnothing$.

For example, the trivial subsequence $S(2 \alpha, 0) \subset S(\alpha, 0)$ has complement $S(2 \alpha,-\alpha)$. Does a nontrivial subsequence of $S(\alpha, 0)$ have a complement with respect to $S(\alpha, 0)$ ?

Theorem 8. For $\alpha, \beta$ of the form (9), (10), $S(\alpha+k \beta, 0)$ (with $k \in\{1, \ldots, t\}$ ), has a complement with respect to $S(\alpha, 0)$ if and only if $t=1$.

Proof. If $S(\eta, w)$ is a complement of $S(\alpha+k \beta, 0)$ with respect to $S(\alpha, 0)$, then a density argument implies $\eta^{-1}+(\alpha+k \beta)^{-1}=\alpha^{-1}$. This has the solution

$$
\eta=\frac{\beta+(k-t) \alpha}{k}
$$

If there is a real number $\gamma$ such that $S(\eta, w) \cup S(\alpha+k \beta, \gamma)=S(\alpha, 0)$ then $\{S(\eta, w), S(\alpha+k \beta, \gamma), S(\beta, 0)\}$ must be a DCS by Proposition I (since $\left.\alpha^{-1}+\beta^{-1}=1\right)$. Consider the three ratios between the three moduli, first

$$
\frac{\alpha+k \beta}{\eta}=\frac{((k+1) \alpha+k t) k}{(k+1-t) \alpha+t} \text {. }
$$

Suppose this ratio is a rational $p / q$. Then $k((k+1) \alpha+k t) q=((k+1-t) \alpha+t) p$, so $k(k+1) q=(k+1-t) p, k^{2} t q=t p$, leading to $k^{2}-t k-1=0$ which has 
the irrational solution $k=\left(t \pm \sqrt{t^{2}+4}\right) / 2$, a contradiction. Hence this ratio is irrational. Also $(\alpha+k \beta) / \beta=\alpha-1+k$ is irrational. Hence the third ratio must be rational by Proposition V. This ratio is

$$
\frac{\beta+(k-t) \alpha}{k \beta}=\frac{1}{k}+\frac{k-t}{k}(\alpha-1),
$$

which is rational if and only if $k=t$. So a necessary condition for a complement to exist is that

$$
\left\{S\left(\frac{\beta}{t}, w\right), S\left(\frac{\beta}{\beta-t-1}, \gamma\right), S(\beta, 0)\right\}
$$

is a DCS, since $\beta /(\beta-t-1)=\alpha+t \beta$. But then Proposition $\mathrm{V}$ implies that $S(\beta, 0) \cup S(\beta / t, w)=S(\beta /(t+1), \delta)$ for some real $\delta$, and $\beta / 2=\beta /(2 t)=$ $\beta /(t+1)$, which implies $t=1$. For $t=1,\{S(\beta /(\beta-2), 0), S(\beta / 2,0)\}$ is indeed a DCS by Proposition IV, and so is

$$
\left\{S\left(\frac{\beta}{\beta-2}, 0\right) S(\beta, 0), S\left(\beta,-\frac{\beta}{2}\right)\right\},
$$

hence $S(\beta /(\beta-2), 0)$ and $S(\beta,-\beta / 2)$ are both nontrivial Beatty subsequences of $S(\alpha, 0)$, and $S(\beta,-\beta / 2)$ is the complement of $S(\beta /(\beta-2), 0)$ with respect to $S(\alpha, 0)$.

\section{ASSOCIATIVITY AND CLOSURE OF SOME BINARY OPERATIONS}

As was stated in $\S 1$, we generalize here a few results from $[19,38]$ to certain infinite classes of algebraic numbers. Many more results can be thus generalized.

For every $m \in \mathbb{Z}$, define $z(m)=\{m \alpha\}$. Note that if $\alpha$ is irrational, then $z$ is $1-1$.

Theorem 9. Let $\alpha$ be a real algebraic integer of degree 2 satisfying

$$
x^{2}+a_{1} x+a_{0}=0 \quad\left(a_{0}, a_{1} \in \mathbb{Z}\right) .
$$

Define

$$
k \otimes l=-a_{1} k l-k A(l)-l A(k) .
$$

Then $z(k \otimes l)=z(k) z(l)$. Thus (i) $k \otimes l$ is associative, (ii) the set of real numbers $z(m)$ is closed under ordinary multiplication.

Proof. By (64),

$$
\begin{aligned}
z(k) z(l)= & (k \alpha-A(k))(l \alpha-A(l)) \\
& =-k l\left(a_{1} \alpha+a_{0}\right)-(k A(l)+l A(k)) \alpha+A(k) A(l) \\
& =r+s \alpha,
\end{aligned}
$$

where

$$
r=A(k) A(l)-a_{0} k l, \quad s=k \otimes l .
$$

Since $0 \leq z(k) z(l)=r+s \alpha<1$, we have

$$
z(k) z(l)=r+s \alpha=\{r+s \alpha\}=\{s \alpha\}=\{(k \otimes l) \alpha\}=z(k \otimes l),
$$

which shows closure. Also (i) follows since $z((k \otimes l) \otimes m)=z(k) z(l) z(m)=$ $z(k \otimes(l \otimes m))$, and since $z$ is $1-1$. 
Corollary 6. $A(k \otimes l)=a_{0} k l-A(k) A(l)$.

Proof. By (66),

$$
0=\lfloor\{s \alpha\}\rfloor=\lfloor\{r+s \alpha\}\rfloor=\lfloor r+s \alpha\rfloor=r+A(s) .
$$

Hence

$$
A(k \otimes l)=A(s)=-r=a_{0} k l-A(k) A(l) .
$$

Lemma 3. For $\alpha, \beta$ of the form (9), (10), $\zeta=\beta-1$ and $p_{i} / q_{i}$ the ith simple continued fraction convergent of $\alpha$, we have

$$
q_{m+1}^{\prime-1}=\left\{(-1)^{m} q_{m} \alpha\right\}=\zeta^{-m-1} \quad(m \geq 0) .
$$

Proof. By (52), $(-1)^{m}\left(q_{m} \alpha-p_{m}\right)=q_{m+1}^{\prime-1}=\left\{(-1)^{m} q_{m} \alpha\right\}$. Note that in the notation of $\S 5$,

$$
\zeta=\beta-1=\left(t+\sqrt{t^{2}+4}\right) / 2=[t, t, t, \ldots]=c_{m+1}^{\prime}
$$

for all $m \geq 0$ is a root of $x^{2}-t x-1=0$. We now show by induction on $m$ that $\zeta q_{m}+q_{m-1}=\zeta^{m+1} \quad(m \geq 0)$. This is seen to hold for $m=0,1$ by (55). If it holds for $m$ and $m-1(m \geq 1)$, then

$$
\begin{aligned}
\zeta q_{m+1}+q_{m} & =\zeta\left(t q_{m}+q_{m-1}\right)+t q_{m-1}+q_{m-2} \\
& =t\left(\zeta q_{m}+q_{m-1}\right)+\left(\zeta q_{m-1}+q_{m-2}\right) \\
& =t \zeta^{m+1}+\zeta^{m}=\zeta^{m}(t \zeta+1)=\zeta^{m+2} .
\end{aligned}
$$

Thus $q_{m+1}^{\prime}=c_{m+1}^{\prime} q_{m}+q_{m-1}=\zeta q_{m}+q_{m-1}=\zeta^{m+1}$.

Theorem 10. For $\alpha, \beta$ of the form (9), (10), $\zeta=\beta-1$ and for every $k, l \in \mathbb{Z}$ and $m \in \mathbb{Z}^{+}$, the diophantine equation

$$
\frac{\{x \alpha\}}{\zeta^{m}}=\frac{\{k \alpha\}}{\zeta^{m}} \frac{\{l \alpha\}}{\zeta^{m}}
$$

has the solution $x=k \otimes l \otimes\left((-1)^{m-1} q_{m-1}\right)$.

Proof. By Lemma 3 and Theorem 9,

$$
\begin{aligned}
\{x \alpha\} & =\{k \alpha\}\{l \alpha\} \zeta^{-m}=\{k \alpha\}\{l \alpha\}\left\{(-1)^{m-1} q_{m-1} \alpha\right\} \\
& =z\left(k \otimes l \otimes\left((-1)^{m-1} q_{m-1}\right)\right) .
\end{aligned}
$$

This suggests to define, for every fixed $m \in \mathbb{Z}^{+}$, a binary operation

$$
k \otimes l=k \otimes\left((-1)^{m-1} q_{m-1}\right) \otimes l .
$$

Theorem 11. For $\alpha, \beta$ of the form (9), (10) and every fixed $m \in \mathbb{Z}^{+}$, the operation $k \cdot l$ is associative, the maps $z_{m}(h)=\{h \alpha\} \zeta^{-m}$ satisfy

$$
z_{m}(k @ l)=z_{m}(k) z_{m}(l)
$$

and the operation $k \Leftrightarrow l$ has the following explicit formula,

$$
\begin{aligned}
k \oplus l=(-1)^{m+1}( & k l q_{m+1}+(k A(l)+l A(k)-2 k l) q_{m} \\
& \left.+(A(k) A(l)-k A(l)-l A(k)+k l) q_{m-1}\right) .
\end{aligned}
$$

Proof. Let $s=k @ l$. Show: $h @ s=(h @ k) @ l$. By Theorem 10,

$$
\frac{z(h(m))}{\zeta^{m}}=\frac{\{h \alpha\}}{\zeta^{m}} \frac{\{s \alpha\}}{\beta^{m}}=\frac{\{h \alpha\}}{\zeta^{m}} \frac{\{k \alpha\}}{\zeta^{m}} \frac{\{l \alpha\}}{\zeta^{m}}=\frac{z((h @ k) \Theta l)}{\zeta^{m}} .
$$


Associativity follows since $z$ is $1-1$. The identity (67) is Theorem 10. For proving the validity of the explicit formula, note that Proposition III implies $A\left((-1)^{m-1} q_{m-1}\right)=(-1)^{m-1} p_{m-1}$. Put $h=k \otimes l$. Then by (65) with $a_{1}=t-2$ (see $(8))$, and by Lemma 2 ,

$$
\begin{aligned}
k @ l & =h \otimes\left((-1)^{m-1} q_{m-1}\right)=(-1)^{m}\left((t-2) h q_{m-1}+h p_{m-1}+A(h) q_{m-1}\right) \\
& =(-1)^{m}\left(h\left(q_{m}-q_{m-1}\right)+A(h) q_{m-1}\right) .
\end{aligned}
$$

Thus by Corollary 6 (with $a_{0}=-t$ ),

$$
\begin{aligned}
k @ l= & (-1)^{m}\left(\left(q_{m}-q_{m-1}\right)(k \otimes l)+A(k \otimes l) q_{m-1}\right) \\
= & (-1)^{m+1}\left(\left(q_{m}-q_{m-1}\right)((t-2) k l+k A(l)+l A(k))\right. \\
& \left.\quad+(t k l+A(k) A(l)) q_{m-1}\right) \\
= & (-1)^{m+1}\left(k l q_{m+1}+(k A(l)+l A(k)-2 k l) q_{m}\right. \\
& \left.\quad+(A(k) A(l)-k A(l)-l A(k)+k l) q_{m-1}\right) .
\end{aligned}
$$

In particular, the operation

$$
k(1) l=k l\left(t^{2}-2 t+2\right)+(k A(l)+l A(k))(t-1)+A(k) A(l)
$$

is associative. This has the simple form $k l+A(k) A(l)$ for $t=1$.

We now turn to mappings which preserve Wythoff pairs. For $\alpha, \beta$ of the form (9), (10) and for every $m \in \mathbb{Z}^{0}$, we call $W(m)=(A(m), B(m))$ a (generalized) Wythoff pair. (For $t=1$, the Wythoff pairs are the second player winning positions of a certain variation of Nim, due to the Dutch mathematician Willem Abraham Wythoff (1885-1939) [1907], who obtained his Ph.D. from the University of Amsterdam in 1898 under Professor Korteweg. The above information is included in bibliographic data on Wythoff which Don Knuth tracked down, via a Dutch friend, "as part of my ongoing search for the full names of people cited in my books", which he kindly communicated to me on December 31, 1990. The generalized Wythoff pairs are, in fact, the second player winning positions of a generalized Wythoff game. See Fraenkel [1982].)

Wythoff pairs will be considered to be either row vectors $(A(m), B(m))$ or column vectors $(A(m), B(m))^{T}$, whatever the context dictates.

Lemma 4. A pair $(a, b) \in\left(\mathbb{Z}^{0}\right)^{2}$ with $0 \leq a \leq b$ is $a$ Wythoff pair if and only if

$$
t \mid(b-a) \text { and } 0 \leq \frac{b-a}{t} \alpha-a<1 .
$$

Proof. Suppose first the conditions hold. By Proposition I $\{(A(m), B(m)): m$ $=1,2, \ldots\}$ is a DCS. Hence we have the following possibilities:

(i) $a=A(k)$ for some $k \in \mathbb{Z}^{0}$,

(ii) $a=B(k)$ for some $k \in \mathbb{Z}^{+}$.

For (i) the divisibility condition and the inequality imply $(b-a) / t=k$, so $(a, b)=(A(k), B(k))$. For (ii) if $l=(b-a) / t$, then $0 \leq l \alpha-B(k)<1$, so $A(l)=B(k)$. By Proposition I this implies $l \leq 0$, so also $\bar{k} \leq 0$, contradicting $k \in \mathbb{Z}^{+}$.

Secondly, assume $(a, b)=(A(m), B(m))$ is a Wythoff pair. Then it is immediate that the two conditions hold. 
Theorem 12. An affine map $T:\left(\mathbb{Z}^{0}\right)^{2} \rightarrow\left(\mathbb{Z}^{0}\right)^{2}$ sends Wythoff pairs into Wythoff pairs if and only if it has the form

$$
T X=\left(\begin{array}{cc}
f & g \\
g & f+g t
\end{array}\right) X+Y=Z, \quad Y=(a, b), \quad f, g \in \mathbb{Z},
$$

where $X=W(m), Z=W(l)$ and $t \mid(b-a)$ imply that $Y$ itself is $a$ Wythoff pair $Y=W(k)$ with

$$
0 \leq f-g(\alpha-1)+\{k \alpha\} \leq 1,
$$

and

$$
l=g A(m)+(f+(t-1) g) m+k .
$$

Proof. Suppose the affine map $T$ sends Wythoff pairs into themselves. That is,

$$
\left(\begin{array}{ll}
f & g \\
i & j
\end{array}\right)\left(\begin{array}{l}
A(m) \\
B(m)
\end{array}\right)+\left(\begin{array}{l}
a \\
b
\end{array}\right)=\left(\begin{array}{l}
A(l) \\
B(l)
\end{array}\right)
$$

i.e.,

$$
f A(m)+g B(m)+a=A(l), \quad i A(m)+j B(m)+b=B(l) .
$$

Subtracting and dividing by $t$,

$$
l=\frac{i+j-f-g}{t} A(m)+(j-g) m+\frac{b-a}{t} .
$$

This identity implies that $(i+j-f-g) A(m) / t$ is an integer for all $m \in$ $\mathbb{Z}^{0}$, hence $(i+j-f-g) / t$ is an integer. Applying identity (11) with $K=$ $(i+j-f-g) / t, L=j-g, M=(b-a) / t$, we get

$$
\begin{gathered}
A(l)=\left(f-i+2(i+j-f-g) t^{-1}\right) A(m)+(i+j-f-g) m+D, \\
D=\left\lfloor\frac{b-a}{t} \alpha+\left(j-g-(i+j-f-g) \alpha^{-1}\right)\{m \alpha\}\right\rfloor .
\end{gathered}
$$

Identifying this expression for $A(l)$ with that of (70) yields

$$
\left(2(i+j-f-g) t^{-1}-i-g\right) A(m)+(i+j-f-g-g t) m+D-a=0 .
$$

Since $A(m) / m \rightarrow \alpha$ as $m \rightarrow \infty$ and $\{m \alpha\}$ varies boundedly with $m$, we get, after dividing by $m$ and letting $m \rightarrow \infty$,

$$
\left(2(i+j-f-g) t^{-1}-i-g\right) \alpha+i+j-f-g-g t=0, \quad D=a .
$$

Since $\alpha$ is irrational, we thus have

$$
i+j=f+g+g t, \quad \frac{2(i+j)}{t}-i=\frac{2(f+g)}{t}+g .
$$

Solving these equations gives

$$
i=g, \quad j=f+g t,
$$

so the matrix has the specified form. Moreover,

$$
a \leq \frac{b-a}{t} \alpha+\left(f+g(t-1)-g t \alpha^{-1}\right)\{m \alpha\}<a+1
$$

so by $(8)$,

$$
0 \leq \frac{b-a}{t} \alpha+(f-g(\alpha-1))\{m \alpha\}-a<1 .
$$


Since this inequality has to hold for all $m \in \mathbb{Z}^{0}$ and since $\{m \alpha\}$ is dense in $[0,1)$, it follows that we have

$$
0 \leq \frac{b-a}{t} \alpha-a+f-g(\alpha-1) \leq 1, \quad 0 \leq \frac{b-a}{t} \alpha-a<1 .
$$

The second of these inequalities and Lemma 4 imply $(a, b)=(A(k), B(k))$ for some $k \in \mathbb{Z}^{0}$. Thus the first inequality becomes (68) as required. Substituting the values of $i, j, a, b$ into (71) gives (69).

Conversely, if the affine map $T$ has the form

$$
\left(\begin{array}{cc}
f & g \\
g & f+g t
\end{array}\right) W(m)+W(k)=Z
$$

subject to (68) where $Z=\left(z_{1}, z_{2}\right)$, then a straightforward computation shows that

$$
t \mid\left(z_{2}-z_{1}\right), \quad \frac{z_{2}-z_{1}}{t} \alpha-z_{1}=(f-g(\alpha-1))\{m \alpha\}+\{k \alpha\},
$$

so by (68) and Lemma 4 it follows that $Z=W(l)$, where $l$ is given by (69).

Corollary 7. For any fixed $\alpha, \beta$ of the form (9), (10), there exist two infinite collections of infinite sets $W_{1}, W_{2}, \ldots$ and $V_{1} \subset V_{2} \subset \cdots$ of Wythoff pairs with the property that $P_{1}+P_{2}$ is a Wythoff pair for every $P_{1} \in W_{i}$ and $P_{2} \in V_{i}$ $(i=1,2, \ldots)$.

Proof. By (52), the odd convergents $f_{i} / g_{i}=p_{2 i+1} / q_{2 i+1} \quad(i \geq 0)$ of $\alpha-1$ satisfy

$$
0<f_{i}-g_{i}(\alpha-1)<g_{i}^{-1}<1 .
$$

Pick any $\left(f_{i}, g_{i}\right)$ of this form. The transformation $T$ with $f=f_{i}, g=g_{i}$ and $k=0$, applied to all Wythoff pairs $X(m)=(A(m), B(m)), m \in \mathbb{Z}^{0}$, produces an infinity $W_{i}$ of Wythoff pairs by Theorem 12 . Since $\{k \alpha\}$ is dense in $[0,1)$, there exists an infinite set $K_{i}$ of $k$ such that (68) holds with $f=f_{i}, g=g_{i}$ and $k \in K_{i}$. The infinite set $V_{i}$ of Wythoff pairs $(A(k), B(k))$ with $k \in K_{i}$ can be added to $W_{i}$ to form a set $Z$ of Wythoff pairs by Theorem 12. Since $g_{i+1}>g_{i}$, it follows that $K_{i+1} \supset K_{i}$, hence $V_{i+1} \supset V_{i}$.

The results of this paper suggest, among other things, the following further research directions.

1. The term "chaos" was used here in a weak technical sense. How "truly chaotic" are sequences such as (34) and (35)? Can the aperiodicity result of Theorem 4 be strengthened to extend to all $k \geq 1$ ? Investigate whether the sequences are pseudo-random. Study the gaps between adjacent points of the sequences for $m=1,2, \ldots, M$ for every finite $M$.

2. Generate chaotic sequences such as (34) and (35) for more general words over the alphabet $\{A, B\}$. This leads also to generalizations of regular sequences of the form (46) and (47).

3. Generalize the results of nonchaotic subsequences of chaotic sequences: (i) Extend Theorem 6 by considering $(A B)^{k}-(B A)^{k}$ for every $k \in$ $\mathbb{Z}^{+}$. This may yield more exact information on expressions of the form $\delta B(A B)^{i}(m)$, at least for infinite subsets $\left\{m_{i}\right\} \subset \mathbb{Z}^{+}$, and thus shed some light on 1 above. (ii) Generalize Theorem 6 to the sequence of the 
denominators of the quasiconvergents $q_{m}=d q_{m-1}+q_{m-2}, 0 \leq d \leq c_{m}$, which, for $m$ even, have properties very similar to $q_{2 m}$, and for $m$ odd, properties similar to $q_{2 m+1}$. See e.g. $[27, \S 9.4$, Ex. 10] or $[37, \S 16$, Nebennäherungsbrüche].

4. The nontrivial Beatty subsequences in $\S 6$ were all subsequences of $S(\alpha, 0)$ with $1<\alpha<2$. Are there also nontrivial Beatty subsequences of $S(\beta, 0)$, where $\beta^{-1}=1-\alpha^{-1}$ ?

5. Complete the generalization of the selection of results from $[19,20,38]$ done in $\S 7$ above, to all results given in these references. For example, generalize the results of [20] to PV numbers of degree $\geq 3$.

6. Which of the results can be extended to larger classes of algebraic numbers, and even to classes of transcendental numbers, and which cannot be extended? Find applications of the basic identities (2) and (5) to algebraic numbers of degree $\geq 3$.

\section{REFERENCES}

1. Th. Bang, On the sequence $[n \alpha], n=1,2, \ldots$, Math. Scand. 5 (1957), 69-76.

2. S. Beatty, Problem 3173, Amer. Math. Monthly 33 (1926), 159; 34 (1927), 159.

3. M. A. Berger, A. Felzenbaum, and A. S. Fraenkel, A non-analytic proof of the Newman-Znám result for disjoint covering systems, Combinatorica 6 (1986), 235-243.

4. _ Disjoint covering systems of rational Beatty sequences, J. Combin. Theory Ser. A 42 (1986), 150-153.

5. J. Berstel, Langford strings are squarefree, Bull. EATCS 37 (1989), 127-129.

6. M. Boshernitzan and A. S. Fraenkel, Nonhomogeneous spectra of numbers, Discrete Math. 34 (1981), 325-327.

7. __ A linear algorithm for nonhomogeneous spectra of numbers, J. Algorithms 5 (1984), 187-198.

8. L. Carlitz, R. Scoville, and V. E. Hoggatt, Fibonacci representations, Fibonacci Quart. 10 (1972), 1-28.

9. L. Dorst and A. W. M. Smeulders, Discrete representation of straight lines, IEEE Trans. Pattern Anal. Machine Intell. PAMI-6 (1984), 450-463.

10. R. B. Eggleton, A. S. Fraenkel, and R. J. Simpson, Beatty sequences and Langford sequences, Discrete Math. 111 (1993), 165-178.

11. P. Erdös, On a problem concerning covering systems, Mat. Lopok 3 (1952), 122-128. (Hungarian; English summary)

12. P. Erdös and R. L. Graham, Old and new problems and results in combinatorial number theory, Monographie No. 28 de L'Enseignement Math., Université de Genève, 1980, 128 pages.

13. A. S. Fraenkel, The bracket function and complementary sets of integers, Canad. J. Math.. 21 (1969), 6-27.

14. $\ldots$, Complementing and exactly covering sequences, J. Combin. Theory Ser. A 14 (1973), 8-20.

15. __ How to beat your Wythoff games' opponent on three fronts, Amer. Math. Monthly 89 (1982), 353-361.

16. __ Systems of numeration, Amer. Math. Monthly 92 (1985), 105-114.

17. A. S. Fraenkel, J. Levitt, and M. Shimshoni, Characterization of the set of values $f(n)=$ [no], $n=1,2, \ldots$, Discrete Math. 2 (1972), 335-345.

18. A. S. Fraenkel, M. Mushkin, and U. Tassa, Determination of $[n \theta]$ by its sequence of differences, Canad. Math. Bull. 21 (1978), 441-446. 
19. A. S. Fraenkel, H . Porta and K. B. Stolarsky, Some arithmetical semigroups, Analytic Number Theory, Proc. of Conf. in Honor 'st Paul T. Bateman (B. C. Berndt, H. G. Diamond, H. Halberstam, and A. Hildebrand, eds.), Progress in Math., vol. 85, Birkhäuser, Boston, Mass., 1990, pp. 255-264.

20. _ _ The almost PV behavior of some far from PV algebraic integers, (1991), Discrete Math, (inpress)..

21. S. W. Golomb, Discrete chaos: sequences satisfying "strange" recursions, (1991), preprint.

22. R. L. Graham, Covering the integers by disjoint sets of the form $\{[n \alpha+\beta]: n=1,2, \ldots\}$, J. Combin. Theory Ser. A 15 (1973), 354-358.

23. R. L. Graham, S. Lin, and C.-S. Lin, Spectra of numbers, Math. Mag. 51 (1978), 174-176.

24. R. K. Guy, Unsolved problems in number theory, Springer-Verlag, New York, 1981.

25. G. H. Hardy and E. M. Wright, An introduction to the theory of numbers, Oxford Univ. Press, 4th ed., Oxford, 1960.

26. D. Hofstadter, Gödel, Escher, Bach, an external golden braid, Random House, New York, 1979.

27. W. J. LeVeque, Fundamentals of number theory, Addison-Wesley, Reading, Mass., 1977.

28. M. Lindenbaum and A. Bruckstein, On recursive $O(N)$ partitioning of a digital curve into digital straight lines (submitted).

29. W. F. Lunnon and P. A. B. Pleasants, Quasicrystallographic tilings, J. Math. Pures Appl. 66 (1987), 217-263.

30. C. L. Mallows, Conway's challenge sequence, Amer. Math. Monthly 98 (1991), 5-20.

31. M. D. Mcllroy, A note on discrete representation of lines, AT\&T Tech. J. 64 (1984), 481-490.

32. F. Mignosi, On the number of factors of Sturmian words, Theoret. Comput. Sci. 82 (1991), 71-84.

33. R. Morikawa, Disjoint sequences generated by the bracket function, Bull. Faculty Liberal Arts, Nagasaki Univ. 26 (1985), 1-13.

34. C. D. Olds, Continued fractions, Random House, New York, 1963.

35. R. Penrose, The role of aesthetics in pure and applied mathematical research, Bull. Inst. Math. Appl. 10 (1974), 266-271.

36. __ Pentaplexity: a class of non-periodic tilings of the plane, Eureka 39 (1978). Reprinted in Math. Intelligencer 2 (1979), 32-37.

37. O. Perron, Die Lehre von den Kettenbrüchen, Band I, Teubner, Stuttgart, 1954.

38. H. Porta and K. B. Stolarsky, Wythoff pairs as semigroup invariants, Adv. in Math. 85 (1991), 69-82.

39. S. Porubský, Results and problems on covering systems of residue classes, Mitt. Math. Sem. Giessen, Heft 150, Giessen, 1981.

40. A. Rosenfeld, Digital straight line segments, IEEE Trans. Comput. C-23 (1974), 1264-1269.

41. R. J. Simpson, The Japanese remainder theorem, Tech. Report 3/90, School of Mathematics and Statistics, Curtin University of Technology, Perth, Western Australia, 1990.

42. $\ldots$, Disjoint covering systems of rational Beatty sequences, Discrete Math. 92 (1991), 361-369.

43. Th. Skolem, On certain distributions of integers in pairs with given differences, Math. Scand. 5 (1957), 57-68.

44. __ Über einige Eigenschaften der Zahlenmengen $[\alpha n+\beta]$ bei irrationalem $\alpha$ mit einleitenden Bemerkungen über einige kombinatorische Probleme, Norske Vid. Selsk. Forh. (Trondheim) 30 (1957), 118-125.

45. N. J. A. Sloane, $A$ handbook of integer sequences, Academic Press, New York, 1973.

46. K. B. Stolarsky, Beatty sequences, continued fractions, and certain shift operators, Canad. Math. Bull. 19 (1976), 473-482.

47. V. Thébault, Les récréations mathématiques, Gauthier-Villars, Paris, 1952.

48. W. D. Wei and C. L. Liu, On a periodic maintenance problem, Oper. Res. Lett. 2 (1983), 90-93. 
49. W. A. Wythoff, A modification of the game of Nim, Nieuw Arch. Wisk. 7 (1907), 199-202.

50. S. Znám, A survey of covering systems of congruences, Acta Math. Univ. Comenian. 40-41 (1982), 59-78.

Department of Applied Mathematics \& Computer Science, The Weizmann Institute of SCIENCE, REHOVOT 76100, ISRAEL

E-mail address: fraenkelerisdom.veizmann.ac.il 